\title{
Aerodynamic Computations Using the Convective-Upstream Split-Pressure Scheme with Local Preconditioning
}

\author{
M. Nemec* and D. W. Zingg ${ }^{\dagger}$ \\ University of Toronto, Toronto, Ontario M3H 5T6, Canada
}

\begin{abstract}
The implementation of the convective-upstream-split-pressure (CUSP) approach to numerical dissipation is presented for an approximately factored algorithm in conjunction with time-derivative local preconditioning. An inexpensive flux limiter is used to blend the low- and high-order CUSP dissipation to capture shocks without oscillations. The resulting algorithm is applied to several subsonic and transonic turbulent aerodynamic flows and compared with results computed using the matrix dissipation scheme. Grid convergence studies are used to assess global errors. The results show the CUSP scheme to be very effective in providing good shock capturing, low numerical dissipation in boundary layers, and low numerical errors. For the flow regimes studied, accuracy is not significantly compromised when the limiter is based on the pressure variable only, leading to significant savings in computational expense. For freestream Mach numbers below 0.2, the convergence rate and accuracy of the solver are significantly improved by preconditioning the CUSP scheme. Overall, the CUSP scheme provides accuracy similar to that of matrix dissipation at a reduced computational cost.
\end{abstract}

\section{Introduction}

$\mathbf{N}$ UMERICAL dissipation is a necessary evil in practical computations of aerodynamic flows, that is, the difference approximation for the convective fluxes cannot be purely skew symmetric The purpose of the symmetric, or dissipative, component of the difference approximationis to produce stable oscillation-freesolutions without significantly compromising solution accuracy, while minimizing the cost of the algorithm. A numerical dissipation scheme has three components: 1) a basic symmetric operator applicable to scalar flux functions, 2) a flux splitting that ensures that all waves are appropriately dissipated when the preceding operator is applied to a hyperbolic system of equations, and 3) some sort of flux limiting or switch to avoid oscillations near shock waves and other discontinuities without contaminating the solution in smooth regions of the flow. The strategies chosen for each of these components can greatly influence the effectiveness and cost of the scheme.

During the early 1980s, the most popular numerical dissipation scheme in aerodynamic computations was the scalar scheme of Jameson et al. ${ }^{1}$ [referred to as the Jameson, Schmidt, and Turkel (JST) scheme], coupled with a pressure switch for shock detection. The JST scheme is inexpensive and robust, but tends to be excessively dissipative in boundary layers., ${ }^{2,3}$ Later in the decade, more sophisticated high-resolution schemes gained in popularity, primarily as a result of their improved discontinuity capturing capabilities. These include flux-vector and flux-difference split upwind schemes ${ }^{4,5}$ and the matrix dissipation scheme, ${ }^{6}$ possibly combined with flux limiters based on total variation diminishing (TVD) concepts. ${ }^{7}$ Typically, high-resolution schemes are more expensive pergrid node than the JST schemebut are usually more cost-effective as a result of their improved accuracy.

More recently, Jameson ${ }^{8,9}$ introduced the convective upstream split pressure (CUSP) scheme and the symmetric limited positive (SLIP) formulation for the construction of high-resolution schemes. The goal of the CUSP scheme is to provide the accuracy of a high-resolution scheme at a computational cost comparable to the JST scheme. There are two versions, ECUSP, and a variation that

Received 11 May 1998; presented as Paper 98-2444 at the AIAA 29th Fluid Dynamics Conference, Albuquerque, NM, 15-18 June 1998; revision received 4 February 1999; accepted for publication 17 August 1999. Copyright (C) 1999 by M. Nemec and D. W. Zingg. Published by the American Institute of Aeronautics and Astronautics, Inc., with permission.

${ }^{*}$ Graduate Student, Institute for Aerospace Studies, 4925 Dufferin Street; marian@oddjob.utias.utoronto.ca.

${ }^{\dagger}$ Professor, Institute for Aerospace Studies, 4925 Dufferin Street. Member AIAA. admits isenthalpic steady solutions, denoted HCUSP. Initial results for flat-plate laminar boundary layers and limited subsonic and transonic airfoil computations presented by Tatsumi et al. ${ }^{10,11}$ were encouraging. Furthermore, Jiang and Damodaran ${ }^{12}$ used the HCUSP scheme successfully for transonic viscous flow computations with various turbulence models. Also, Sheffer et al. ${ }^{13}$ implemented an earlier version of the CUSP scheme, which is related to the advective upwind splitting method (AUSM) of Liou and Steffen, ${ }^{14}$ and obtained good results for hypersonic reacting flows. However, Swanson et al. ${ }^{15}$ performed an evaluation of the HCUSP scheme and found it to be somewhat less accurate and up to $25 \%$ more expensive than matrix dissipation with a simple pressure switch.

Low-Mach-number flows introduce additional difficulties for compressible flow solvers as a result of the wide range of wave speeds associated with this flow regime, which can result in poor convergence of an iterative method. ${ }^{16,17}$ In addition, the accuracy of the solver may degrade as the Mach number is reduced because of poor scaling of the numerical dissipation. Local preconditioning techniques have been developed to address these difficulties, leading to greatly improved convergence rates and reduced numerical errors at low Mach numbers. ${ }^{18-22}$ Unrau and Zingg ${ }^{23}$ showed that the accuracy of the JST scheme at low Mach numbers can be greatly improved using the preconditioner of Weiss and Smith. ${ }^{24}$ Tweedt et al. ${ }^{25}$ present results for low-Mach-number turbomachinery flows using a preconditioned scheme, which is equivalent to the CUSP scheme, showing improvements in accuracy and degradation in convergence relative to a preconditioned JST scheme. Edwards and Liou $^{26}$ present extensions of AUSM for use with local preconditioning.

This paper has two objectives. First we present a thorough evaluation of the ECUSP scheme in the context of subsonic and transonic two-dimensionalturbulent flow over airfoils. The ECUSP scheme is compared with the matrix dissipationscheme in terms of dissipation levels in boundary layers, shock-capturing capability, global solution errors, convergence rates, and computational expense. Global errors are assessed through grid-convergence studies. The implications of different flux-limiting options are considered as well, and an inexpensive new approach is proposed. The second objective is to present a locally preconditioned version of the CUSP scheme using the preconditioner of Weiss and Smith ${ }^{24}$ and to demonstrate that at low Mach numbers, the resulting scheme produces convergence rates and solutions which are virtually independentof Mach number.

\section{Governing Equations and Numerical Method}

The matrix and CUSP numerical dissipation schemes have been implemented in the thin-layerNavier-Stokes solver ARC2D. ${ }^{27}$ This 
solver uses second-order centered differences in space through a generalized curvilinear coordinate transformation and is thus applicable to structured grids. Convergence to steady state is achieved using the diagonal form of the Beam and Warming approximate-factorization algorithm with local time stepping. The effects of turbulence are modeled by the Baldwin-Lomax turbulence model. A circulation correction is used at the outer boundary.

In two-dimensional generalized coordinates the thin-layer Navier-Stokes equations are given $\mathrm{by}^{27}$

$$
\frac{\partial \hat{Q}}{\partial t}+\frac{\partial \hat{E}}{\partial \xi}+\frac{\partial \hat{F}}{\partial \eta}=R^{-1} \frac{\partial \hat{S}}{\partial \eta}
$$

where $Q=J^{-1} Q=J^{-1}[\rho, \rho u, \rho v, e]^{T}$ is the vector of conservative dependent variables; $\hat{E}$ and $\hat{F}$ are the convective flux vectors; $\hat{S}$ is the viscous flux vector; $\xi$ and $\eta$ are the streamwise and normal generalized coordinates, respectively; $J$ is the Jacobian of the coordinate transformation; and $R e$ is the freestream Reynolds number based on the speed of sound.

The implementation of local preconditioning into the ARC2D solver is described by Unrau and Zingg ${ }^{23}$ and is based on the work of Weiss and Smith, ${ }^{24}$ which is closely related to that of Choi and Merkle ${ }^{19}$ and Turkel. ${ }^{20,28}$ The preconditioning technique not only accelerates the convergence rate of the solver but also improves the accuracy of the solution for low-Mach-numberflow. The where $\phi=1$ for the presented test cases. Further details of the implementation of local preconditioning, such as the time-step definition and the treatment of the far-field boundary conditions, as well as several results obtained using the JST scheme, are given in Ref. 23.

\section{Numerical Dissipation Schemes}

\section{Matrix Dissipation with Pressure Switch}

Because the matrix numerical dissipation scheme is used for comparison purposes to evaluate the CUSP scheme, we present our implementation here. The matrix scheme is based on that of Swanson and Turkel ${ }^{6}$ and is added to the second-order centered difference scheme in generalized coordinates in the following manner:

$$
\frac{\partial \hat{E}}{\partial \xi} \approx \delta_{\xi} \hat{E}-\nabla_{\xi} D_{1} D_{2}
$$

with

$$
\begin{gathered}
D_{1}=|A|_{j+\frac{1}{2}, k} J_{j+\frac{1}{2}, k}^{-1} \\
D_{2}=\epsilon_{j+\frac{1}{2}, k}^{(2)} \Delta_{\xi} Q_{j, k}-\epsilon_{j+\frac{1}{2}, k}^{(4)} \nabla_{\xi} \Delta_{\xi} \nabla_{\xi} Q_{j, k} \\
\epsilon_{j, k}^{(2)}=\kappa_{2} \Delta t \max \left(\mathrm{Y}_{j+1, k}, \mathrm{Y}_{j, k}, \mathrm{Y}_{j-1, k}\right) \\
\epsilon_{j, k}^{(4)}=\max \left[0, \kappa_{4} \Delta t-\epsilon_{j, k}^{(2)}\right]
\end{gathered}
$$

$$
\mathrm{Y}_{j, k}=\frac{\left|p_{j+1, k}-2 p_{j, k}+p_{j-1, k}\right|}{\left(1-v_{j, k}\right)\left(\left|p_{j+1, k}-p_{j, k}\right|+\left|p_{j, k}-p_{j-1, k}\right|\right)+v_{j, k}\left(p_{j+1, k}+2 p_{j, k}+p_{j-1, k}\right)}
$$

preconditioned form of Eq. (1) is obtained by introducing the preconditioning matrix $\Gamma$ as follows:

$$
\Gamma \frac{\partial \hat{Q}}{\partial t}+\frac{\partial \hat{E}}{\partial \xi}+\frac{\partial \hat{F}}{\partial \eta}=R e^{-1} \frac{\partial \hat{S}}{\partial \eta}
$$

To simplify the form of the preconditioningmatrix, the following symmetry variables are used ${ }^{21}$ :

$$
\partial \widetilde{Q}=J^{-1}\left[\begin{array}{c}
(1 / \rho c) \partial p \\
\partial u \\
\partial v \\
\partial p-c^{2} \partial \rho
\end{array}\right]
$$

The symmetry variables are related to the conservative variables through the transfer matrices given by

$$
M=\frac{\partial \mathscr{Q}}{\partial \widetilde{Q}}, \quad M^{-1}=\frac{\partial \widetilde{Q}}{\partial \mathscr{Q}}
$$

The local preconditioner formulated in terms of the symmetry variables can be applied to the thin-layer Navier-Stokes equations written in terms of the conservative variables as follows ${ }^{23}$ :

$$
M \tilde{\Gamma} M^{-1} \frac{\partial \hat{Q}}{\partial t}+\frac{\partial \hat{E}}{\partial \xi}+\frac{\partial \hat{F}}{\partial \eta}=R e^{-1} \frac{\partial \hat{S}}{\partial \eta}
$$

The preconditioner of Weiss and Smith, ${ }^{24}$ written in terms of the symmetry variables, has the following form:

$$
\tilde{\Gamma}=\left[\begin{array}{cccc}
1 / \epsilon & 0 & 0 & 0 \\
0 & 1 & 0 & 0 \\
0 & 0 & 1 & 0 \\
0 & 0 & 0 & 1
\end{array}\right]
$$

where

$$
\epsilon=\min \left[1, \max \left(M^{2}, \epsilon_{1}\right)\right]
$$

The parameter $\epsilon_{1}$ is used to ensure that $\epsilon$ remains finite in stagnation regions and is given by

$$
\epsilon_{1}=\phi M_{\infty}^{2}
$$

$$
v_{j, k}=\frac{\min \left(p_{j+1, k}, p_{j, k}, p_{j-1, k}\right)}{\max \left(p_{j+1, k}, p_{j, k}, p_{j-1, k}\right)}
$$

where $\delta_{\xi}$ is a second-order centered difference operator, $\Delta_{\xi}$ and $\nabla_{\xi}$ are first-order forward and backward difference operators, $U$ is a contravariant velocity component, and $\kappa_{2}$ and $\kappa_{4}$ are constants. Typical values of $\kappa_{2}$ and $\kappa_{4}$ are 1.0 and 0.02 , respectively. The term $\mathrm{Y}_{j, k}$ is a TVD pressure switch ${ }^{6,29}$ used to control the first-order dissipation near shock waves.

The matrix $|A|$ is given by

$$
|A|=T_{\xi}|\Lambda|_{\xi} T_{\xi}^{-1}
$$

where the columns of $T_{\xi}$ are the right eigenvectors of $A$, the flux Jacobian, and

$$
|\Lambda|_{\xi}=\left[\begin{array}{cccc}
|U| & 0 & 0 & 0 \\
0 & |U| & 0 & 0 \\
0 & 0 & |U+c \psi| & 0 \\
0 & 0 & 0 & |U-c \psi|
\end{array}\right]
$$

with $\psi=\sqrt{ }\left(\xi_{x}^{2}+\xi_{y}^{2}\right)$. The terms in the $\eta$ direction are analogous.

Near stagnation points and sonic points some of the eigenvalues of $A$ approach zero. To avoid the problems this may introduce, the elements of $|\Lambda|_{\xi}$ can be modified as follows:

$$
\begin{gathered}
\lambda_{1}, \lambda_{2}=\max \left(\lambda_{1,2}, V_{l} \sigma\right), \quad \lambda_{3}=\max \left(\lambda_{3}, V_{n} \sigma\right) \\
\lambda_{4}=\max \left(\lambda_{4}, V_{n} \sigma\right)
\end{gathered}
$$

where $\sigma$ represents the spectral radius of the flux Jacobian. The constants $V_{l}$ and $V_{n}$ can be set to zero for subsonic flows, but finite values are needed for transonic flows. Commonly used values are $V_{l}=0.025$ and $V_{n}=0.25$. The scheme has been implemented using an efficient matrix-vectormultiply technique as presented in Ref. 6.

\section{CUSP Dissipation Scheme}

The CUSP ${ }^{8,9}$ scheme is formulated by a combination of differences of the state and flux vectors. We consider the ECUSP version only and refer to it as the CUSP scheme for the remainder of the paper. 
The first-order CUSP scheme for the $\xi$ coordinate direction is given by

$$
\begin{gathered}
d_{j+\frac{1}{2}, k}=\frac{1}{2} J_{j+\frac{1}{2}, k}^{-1} \alpha_{j+\frac{1}{2}, k}^{\star} c\left(Q_{j+1, k}-Q_{j, k}\right) \\
+\frac{1}{2} J_{j+\frac{1}{2}, k}^{-1} \beta_{j+\frac{1}{2}} \Delta E_{j+\frac{1}{2}, k}
\end{gathered}
$$

where

$$
\Delta E_{j+\frac{1}{2}, k}=\xi_{x_{j+\frac{1}{2}, k}}\left(E_{j+1, k}-E_{j, k}\right)+\xi_{y_{j+\frac{1}{2}, k}}\left(F_{j+1, k}-F_{j, k}\right)
$$

The vectors $E$ and $F$ are the flux vectors written in Cartesian coordinates. The factor $c$ (local speed of sound) is included so that $\alpha^{\star}$ is dimensionless. The parameters $\alpha^{\star}$ and $\beta$ are given by

$$
\begin{aligned}
& \alpha^{\star} c=\alpha c-\beta \bar{U}
\end{aligned}
$$

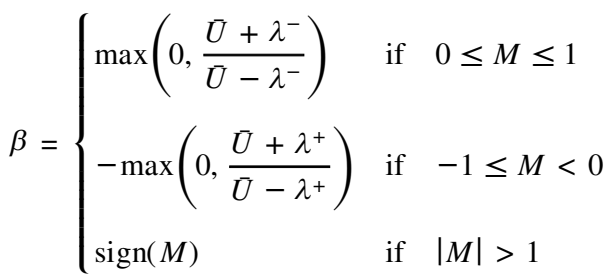

where

$$
\lambda^{ \pm}=U \pm c \sqrt{\xi_{x}^{2}+\xi_{y}^{2}}
$$

and $\alpha$ is chosen to equal $|O| / c$. The symbol $O$ is the arithmetic mean contravariant velocity, whereas the eigenvalues $\lambda^{ \pm}$are determined at the Roe state. For supersonic flows the CUSP scheme becomes an upwind scheme. The CUSP scheme is not local-extremumdiminishing (LED) and does not guarantee an oscillation-free solution. This is discussed in Ref. 15 and shown through a numerical experiment in Ref. 30. However, CUSP has been formulated to produce single-point shocks for inviscid flows (if the Roe average is used in determining the eigenvalues).

This choice of the parameters $\alpha^{\star}$ and $\beta$ means that for Mach numbers below 0.5 the CUSP scheme is a scalar scheme with a scaling based on the contravariant velocity component in the appropriate curvilinear coordinate direction. If the grid is aligned with the flow in a boundary layer, the scaling of the CUSP scheme in the $\eta$ direction is based on $|V|$, the contravariant velocity component in the $\eta$ direction, which can be small, much smaller than the scaling $|V|+c \sqrt{ }\left(\eta_{x}^{2}+\eta_{y}^{2}\right)$ as in the JST scheme. Consequently, the CUSP scheme produces much less dissipation than the JST scheme in boundary layers.

To construct a higher-orderCUSP scheme, limiters are added that activate near flow discontinuities. Jameson ${ }^{8}$ introduced the following limiter function:

$$
R(u, v)=1-\left|\frac{u-v}{|u|+|v|}\right|^{q}
$$

where we have used $q=2$. A higher-orderCUSP scheme is obtained by defining the limited average

$$
L(u, v)=\frac{1}{2} R(u, v)(u+v)
$$

and then constructing the appropriate left and right states for each variable:

$$
\begin{gathered}
Q_{L}^{(n)}=Q_{j}^{(n)}+\frac{1}{2} L\left[\Delta Q_{j+\frac{3}{2}, k}^{(n)}, \Delta Q_{j-\frac{1}{2}, k}^{(n)}\right] \\
Q_{R}^{(n)}=Q_{j+1}^{(n)}-\frac{1}{2} L\left[\Delta Q_{j+\frac{3}{2}, k}^{(n)}, \Delta Q_{j-\frac{1}{2}, k}^{(n)}\right]
\end{gathered}
$$

where the superscript $n$ representsthe $n$th element of the state vector. Consequently, the dissipative flux becomes

$$
\begin{gathered}
d_{j+\frac{1}{2}, k}=\frac{1}{2} J_{j+\frac{1}{2}, k}^{-1} \alpha_{j+\frac{1}{2}, k}^{\star}\left(Q_{R, k}-Q_{L, k}\right) \\
+\frac{1}{2} J_{j+\frac{1}{2}, k}^{-1} \beta_{j+\frac{1}{2}} \Delta E\left(Q_{R, L}{ }_{j+\frac{1}{2}, k}\right)
\end{gathered}
$$

where

$$
\begin{aligned}
& \Delta E\left(Q_{R, L_{j+\frac{1}{2}, k}}\right)=\xi_{x_{j+\frac{1}{2}, k}}\left[E\left(Q_{R, k}\right)-E\left(Q_{L, k}\right)\right] \\
& \quad+\xi_{y_{j+\frac{1}{2}, k}}\left[F\left(Q_{R, k}\right)-F\left(Q_{L, k}\right)\right]
\end{aligned}
$$

Analogous terms appear in the $\eta$ direction.

A modification to the limited average is suggested by Swanson et al. ${ }^{15}$ where a free parameter is introduced to decouple the constant scaling of the first-order dissipation from the constant scaling of the third-order dissipation. We found that for the test cases presented the accuracy of the solution was not significantly influenced by this parameter.

Near a flow discontinuity $u$ and $v$ in Eq. (16) have opposite signs, and therefore $R(u, v) \rightarrow 0$. A problem with this limiter function is that it is insensitive to the relative magnitudes of $u$ and $v$. In near constant regions of the flowfield, small fluctuations in $u$ and $v$ can trigger the limiter randomly, which may stall the convergence of the solution. One way to overcome this difficulty is to freeze the limiter once the convergence rate stalls. A better approach is suggested by Jameson,${ }^{8}$ who follows the work of Venkatakrishnan. ${ }^{31}$ The limiter function is modified as follows:

$$
R(u, v)=1-\left|\frac{u-v}{\max \left(|u|+|v|,(\varepsilon \Delta x)^{\frac{3}{2}}\right)}\right|^{q}
$$

where

$$
\Delta x=\sqrt{x_{\xi}^{2}+x_{\eta}^{2}}
$$

Note that all quantities are nondimensional. This soft limiter introduces a threshold below which first-orderdissipationis not activated, and, therefore, triggering of the limiter for small extrema does not occur. For inappropriate values of $\varepsilon$, the resulting threshold may be too high, and, consequently,visible oscillations may develop around discontinuities.

Venkatakrishnan ${ }^{31}$ suggests values for $\varepsilon$ between 1.0 and 5.0. We use $\varepsilon=5.0$ and refer to this limiter as the $\mathrm{V}$ limiter.

We also modified Eq. (16) to create a new limiter, which is shown next:

$$
R(u, v)=1-\left|\frac{u-v}{|u|+|v|+\varepsilon /(|u|+|v|)}\right|^{q}
$$

In this case the parameter $\varepsilon$ controls the activation of the limiter. For transonic flows appropriate values for $\varepsilon$ are between $10^{-2}-10^{-4}$. We use $\varepsilon=10^{-3}$ and refer to this limiter as the $\mathrm{Z}$ limiter. Note that this limiter requires less expense than the $\mathrm{V}$ limiter, primarily because it avoids the use of the max function.

The use of the limiters defined by Eqs. (16), (20), and (21) adds considerable computational expense to the algorithm. The limiter value has to be computed for each state variable at each node for each direction (i.e., eight evaluations of the limiter function) compared to just two evaluations per node of the pressure switch function. Further, the inversion of the left-hand side of the implicit algorithm becomes less efficient because the limiter may apply different values to each conservation equation. To increase the efficiency of the algorithm, we also consider the use of the pressure variable in the $\mathrm{Z}$ limiter, with the same limiting applied to each conservation equation. We refer to this limiter as the ZP limiter. Its computational expense is equivalent to that of the pressure switch. Its use is recommended only for flows in which discontinuities (or near discontinuities) are characterized by a discontinuous pressure field, as opposed to discontinuities such as contact surfaces through which the pressure is continuous. 
On the left-hand side of the implicit approximately factored algorithm, we use the diagonal form. The eigenvalues associated with the CUSP scheme and the appropriate limiter values are added to the diagonal entries.

\section{Locally Preconditioned CUSP Scheme}

For low-Mach-number flow the CUSP scheme reduces to scalar dissipation with scaling proportional to the contravariant velocity component. Swanson et al. ${ }^{15}$ note that with this scaling CUSP dissipation is well suited for low-Mach-number flow because a similar scaling is used by the preconditioned JST scheme, provided that the dissipation is augmented by the preconditioning matrix.

To demonstrate the necessity of augmenting the CUSP dissipation vector by the preconditioning matrix $\Gamma$, consider the following analysis. We use the one-dimensionalEuler equations to express the order of magnitude of the elements in the $\Gamma$ matrix, state vector, and flux vector in terms of $\epsilon$. Recall that effective low-Mach-number preconditioning is obtained with $\epsilon=M^{2}$, which is given in Eq. (7).

For low-Mach-numberflow the changes in the state vector $q$ scale with $\epsilon$ as follows:

$$
\Delta q=\left[\begin{array}{c}
\Delta \rho \\
\Delta \rho u \\
\Delta e
\end{array}\right]=\left[\begin{array}{c}
\mathcal{O}(\epsilon) \\
\mathcal{O}\left(\epsilon^{\frac{1}{2}}\right) \\
\mathcal{O}(\epsilon)
\end{array}\right]
$$

as can be derived from the isentropic relations. The order of magnitude for a scalar scaling proportional to $\bar{u}$, as used by the CUSP scheme for low Mach numbers, is $\mathcal{O}\left(\epsilon^{1 / 2}\right)$. Therefore, first-order CUSP dissipation can be expressed in terms of the order of $\epsilon$ as follows:

$$
\bar{u} \Delta q=\left[\begin{array}{c}
\mathcal{O}\left(\epsilon^{\frac{3}{2}}\right) \\
\mathcal{O}(\epsilon) \\
\mathcal{O}\left(\epsilon^{\frac{3}{2}}\right)
\end{array}\right]
$$

The order of the dissipation vector should be the same as the flux vector. The order of magnitude for the flux vector is

$$
\Delta f=\left[\begin{array}{c}
\Delta \rho u \\
\Delta\left(\rho u^{2}+p\right) \\
\Delta u(e+p)
\end{array}\right]=\left[\begin{array}{c}
\mathcal{O}\left(\epsilon^{\frac{1}{2}}\right) \\
\mathcal{O}(\epsilon) \\
\mathcal{O}\left(\epsilon^{\frac{1}{2}}\right)
\end{array}\right]
$$

Comparing Eq. (24) with Eq. (23) reveals that the mass and energy conservation equations have insufficient numerical dissipation at low Mach numbers, which can cause problems with stability and convergence.

To make the order of the dissipation vector consistent with the flux vector, consider the $\Gamma$ matrix whose entries in terms of the order of $\epsilon$ are

$$
\Gamma=\left[\begin{array}{ccc}
\mathcal{O}(1) & \mathcal{O}\left(\epsilon^{-\frac{1}{2}}\right) & \mathcal{O}\left(\epsilon^{-1}\right) \\
\mathcal{O}(\epsilon) & \mathcal{O}(1) & \mathcal{O}\left(\epsilon^{-\frac{1}{2}}\right) \\
\mathcal{O}(1) & \mathcal{O}\left(\epsilon^{-\frac{1}{2}}\right) & \mathcal{O}\left(\epsilon^{-1}\right)
\end{array}\right]
$$

Then the product $\Gamma \Delta q$ becomes

$$
\Gamma \Delta q=\left[\begin{array}{c}
\mathcal{O}(1) \\
\mathcal{O}\left(\epsilon^{\frac{1}{2}}\right) \\
\mathcal{O}(1)
\end{array}\right]
$$

Now, if the dissipation vector is augmented by the preconditioning matrix in the following manner:

$$
\bar{u} \Gamma \Delta q=\left[\begin{array}{c}
\mathcal{O}\left(\epsilon^{\frac{1}{2}}\right) \\
\mathcal{O}(\epsilon) \\
\mathcal{O}\left(\epsilon^{\frac{1}{2}}\right)
\end{array}\right]
$$

the order of the dissipation vector is consistent with the order of the flux vector.
Hence, to obtain the preconditionedCUSP scheme for flow with freestream Mach number below 0.5, modify Eq. (13) as follows:

$$
d_{j+\frac{1}{2}, k}=\frac{1}{2} \alpha_{j+\frac{1}{2}, k}^{\star} c \Gamma_{j+\frac{1}{2}, k} J_{j+\frac{1}{2}, k}^{-1}\left(Q_{j+1, k}-Q_{j, k}\right)
$$

where

$$
\alpha^{\star}=\bar{U} / c
$$

Both $\mathrm{V}$ and $\mathrm{Z}$ limiters can be used with the scheme; however, the subsonic test case studied did not require these limiters.

\section{Results and Discussion}

To evaluate the CUSP numerical dissipation scheme, the following test cases are examined:

1) $M_{\infty}=0.16, \alpha=0 \mathrm{deg}, \operatorname{Re}=2.88 \times 10^{6}$, transition at 0.43 chords on both surfaces, NACA 0012 airfoil.

2) $M_{\infty}=0.16, \alpha=6 \mathrm{deg}, R e=2.88 \times 10^{6}$, transition at 0.05 and 0.8 chords on the upper and lower surfaces, respectively, NACA 0012 airfoil.

3) $M_{\infty}=0.7, \alpha=3 \mathrm{deg}, \operatorname{Re}=9.0 \times 10^{6}$, transition at 0.05 chords on both surfaces, NACA 0012 airfoil.

4) $M_{\infty}=0.729, \alpha=2.31 \mathrm{deg}, R e=6.5 \times 10^{6}$, transition at 0.03 chords on both surfaces, RAE 2822 airfoil.

5) $M_{\infty}=0.01,0.05,0.1,0.2,0.3,0.4 ; \alpha=2.0 \mathrm{deg} ; R e=2.88 \times$ $10^{6}$; transition at 0.05 and 0.25 chords on the upper and lower surfaces, respectively, NACA 0012 airfoil.

Table 1 is a summary of the grids used. All of the grids have a $\mathrm{C}$ topology. In Table $1 \mathrm{JDIM}$ represents the number of points in the streamwise direction, KDIM represents the number of points in the normal direction, Body points represents the number of points on the body, Wake points represents the number of points in the wake, and Off-wall spacing is the normal spacing to the first grid line off of the surface in chords. The distance to the outer boundary is 12 chords for all grids. For grid A the leading-edgeclustering is 0.0001 , and the trailing-edgeclustering is 0.0002 . Grid B was generated by removing every second node in both coordinate directions from grid $\mathrm{A}$, and similarly grid $\mathrm{C}$ was generated by removing every second node in both coordinate directions from grid $\mathrm{B}$. Grid $\mathrm{P}$ is used for case 5 . Note that grid A, which has roughly 200,000 nodes, is much finer than grids generally used in practice. As we will see next, results for grid $\mathrm{A}$ are approaching grid independence and thus can be used as a benchmark to estimate solution errors.

\section{Dissipation in Boundary Layers}

We first examine the amount of numerical dissipation introduced in boundary layers, and whether it interferes with the desired balance between convective and viscous fluxes. The streamwise momentum equation is typically most revealing. ${ }^{3}$ Figure 1 shows the $x$-momentum flux balance for case 1 computed on grid $\mathrm{C}$ with the matrix and CUSP schemes. The user-selected parameters for matrix dissipation were set to $\kappa_{2}=0$ and $\kappa_{4}=0.02$ with $V_{n}=V_{l}=0$. For CUSP dissipation the limiters were not activated. The station shown is at $x / c=0.6$, where the boundary-layerthickness is roughly 0.008 chords and the flow is turbulent. There are roughly 25 points across the boundary layer. Ideally, the viscous flux should balance the convective flux with only a minimal contribution from the numerical dissipation. This is well illustrated by both schemes in Fig. 1. Consequently, the solutions obtained by the matrix and CUSP schemes are very similar. Even on the coarser grid $\mathrm{C}$, the drag coefficient was within $1.5 \%$ of the drag coefficient computed on grid $\mathrm{A}$ for both schemes.

Table 1 Summary of grids

\begin{tabular}{lccccc}
\hline \hline Grid & JDIM & KDIM & $\begin{array}{c}\text { Body } \\
\text { points }\end{array}$ & $\begin{array}{c}\text { Wake } \\
\text { points }\end{array}$ & $\begin{array}{c}\text { Off-wall } \\
\text { spacing }\end{array}$ \\
\hline A & 1057 & 193 & 801 & 129 & $2.3 \times 10^{-7}$ \\
B & 529 & 97 & 401 & 65 & $5.3 \times 10^{-7}$ \\
C & 265 & 49 & 201 & 33 & $1.2 \times 10^{-6}$ \\
P & 279 & 49 & 201 & 40 & $2 \times 10^{-6}$ \\
\hline \hline
\end{tabular}




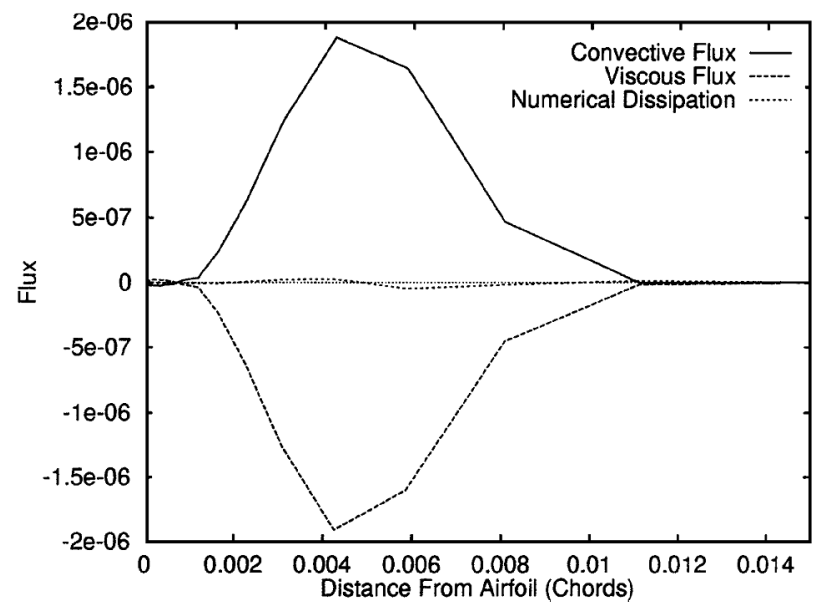

Matrix dissipation

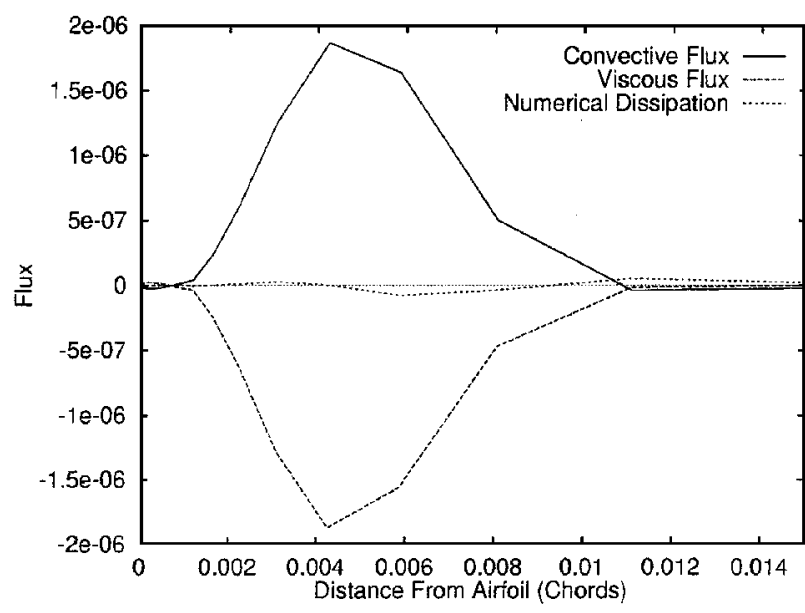

CUSP dissipation

Fig. 1 Flux balance for case 1 on grid $\mathrm{C}$ at $60 \%$ chord.

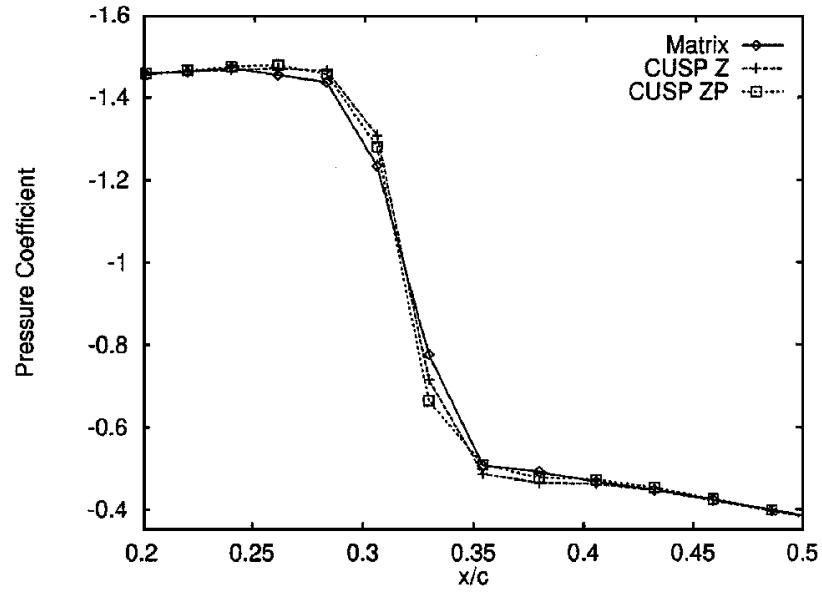

a) Shock region for case 3

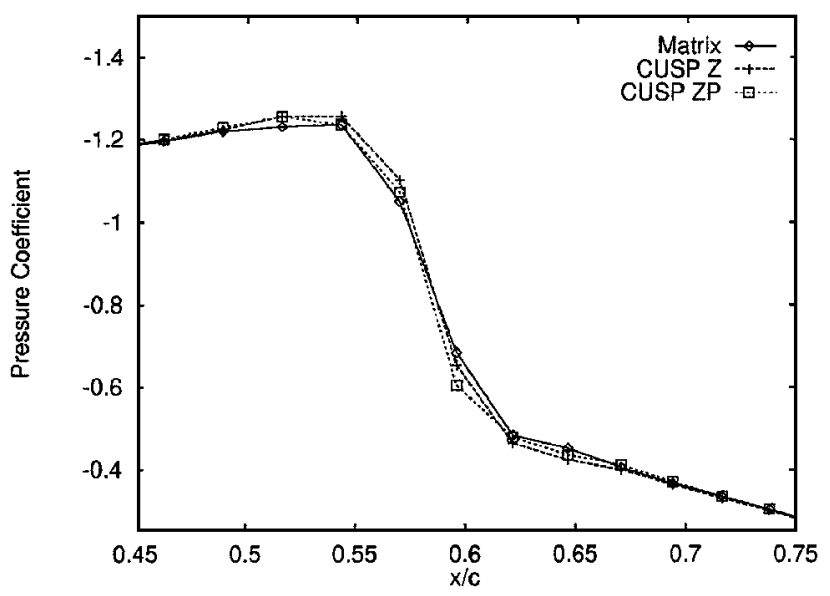

b) Shock region for case 4

Fig. 2 Computed pressure distributions around the shock region for cases 3 and 4 on grid $C$.

\section{Shock Capturing}

Both matrix and CUSP dissipation capture the shock very well as shown in Figs. 2a and 2b. The quality of shock capture for the pressure-based ZP limiter is approximately the same as for the Z limiter, but the computational expense of the algorithm is significantly reduced. Note that single-point shocks are obtained when using the CUSP scheme for inviscid flows if the Roe average is used in computing the eigenvalues.

\section{Accuracy}

Figures 3-5 show the grid convergence of the lift coefficient $C_{l}$, drag coefficient $C_{d}$, the pressure drag component $C_{d_{p}}$, and the friction drag component $C_{d_{f}}$ for cases 2,3 , and 4 . The results are plotted vs $1 / N$, where $N$ is the total number of grid nodes. In all cases the percent difference between solutions on successive grids decreases with refinement, indicating that the grid A solutions approach grid independence. For example, in Fig. 3 the difference between $C_{d}$ computed on grids A and B is roughly $3 \%$, whereas grids $\mathrm{B}$ and $\mathrm{C}$ differ by $12 \%$. These figures show that overall the errors obtained using CUSP are similar in magnitude to those obtained using matrix dissipation.Use of the JST scheme leads to much larger errors. ${ }^{3}$ Matrix dissipation is slightly more accurate in predicting the lift coefficient and the friction drag coefficient. The CUSP scheme produces more accurate pressure drag values for the transonic cases. Note that error cancellation between the $C_{d_{p}}$ and $C_{d_{f}}$ values may influence the global $C_{d}$ error. Results computed with the less expensive ZP limiter are very similar to those obtained using the full $\mathrm{Z}$ limiter.

\section{Computational Expense}

The residual convergencehistories for cases 2 and 4 are shown in Fig. 6. Similar convergence rates are obtained using the CUSP and matrix dissipation schemes. The matrix scheme with the pressure switch requires 15-20\% more computationaleffort per iteration than the JST scheme, depending on the details of the implementation, primarily as a result of the need to form the necessary matrix-vector products. The CUSP scheme with the ZP limiter has a computational cost only 5-10\% greater than that of the JST scheme. The cost is associated with the use of the Roe average, which is not strictly necessary for the class of flows considered here. With the Z limiter the CUSP scheme requires about $35 \%$ more effort than the JST scheme and with the V limiter about $40 \%$, which is because of the evaluation of the limiter. Clearly the use of the simple pressurebased limiter provides significant savings for the flow regimes under consideration here.

\section{Local Preconditioning}

Results for case 5 are presented in Fig. 7, which shows the variation of the computed drag with the freestream Mach number for the CUSP scheme on grid $\mathrm{P}$ with and without local preconditioning. The original CUSP scheme without preconditioning shows a dependence on the Mach number for Mach numbers less than 0.2. The preconditioned CUSP scheme correctly produces drag coefficients that are independent of the Mach number for the given Mach number range.

Figure 8 shows the number of iterations required to converge the lift and drag to within $0.1 \%$ of their converged values as a function of the freestream Mach number. With local preconditioning 

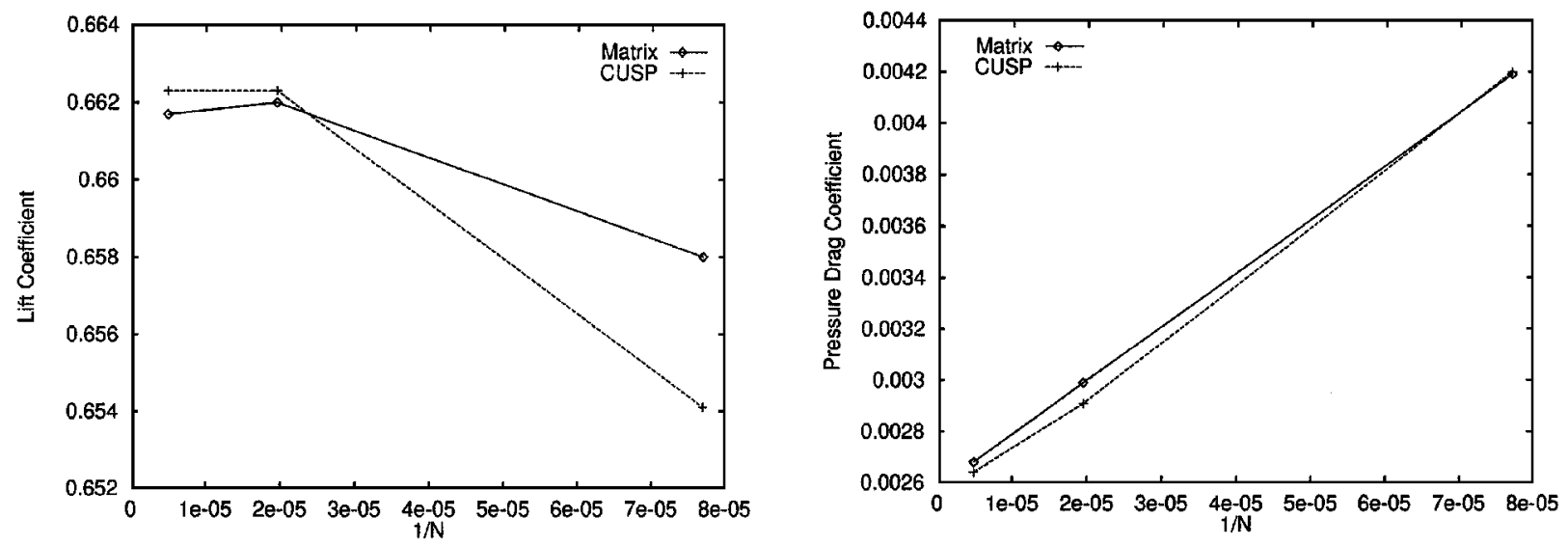

$C_{l}$

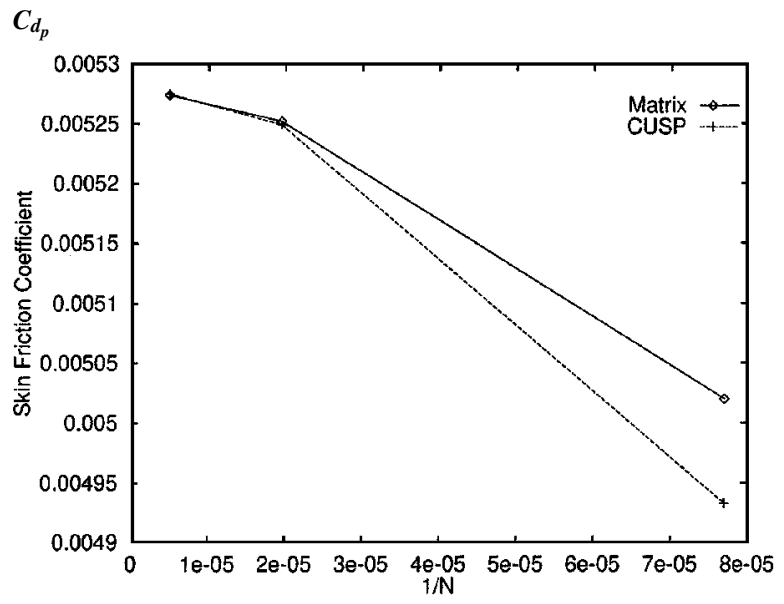

$C_{d}$

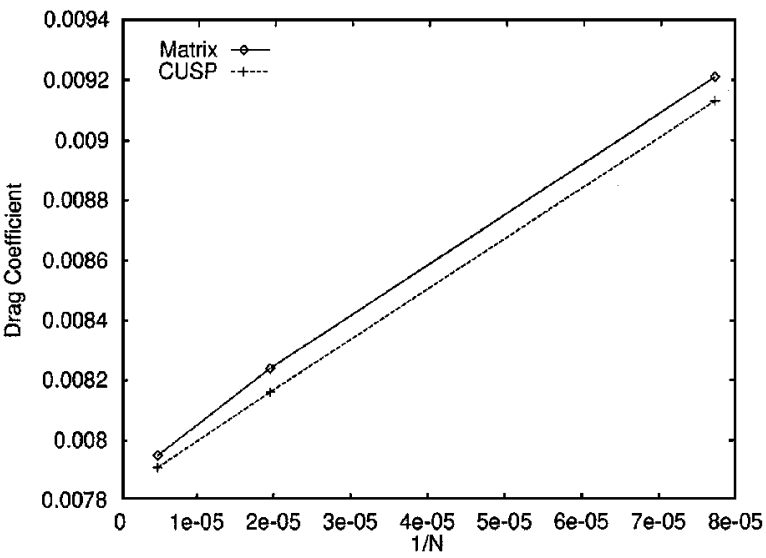

$\boldsymbol{C}_{d_{f}}$

Fig. 3 Grid convergence for case 2.
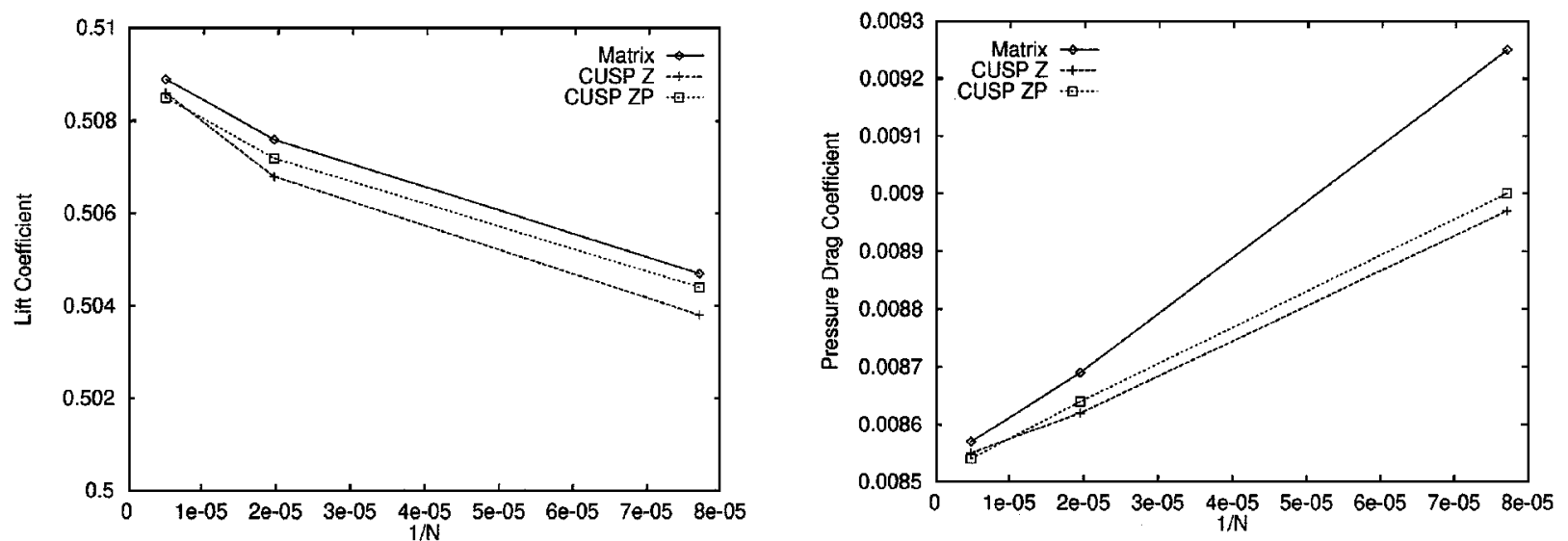

$C_{l}$

$C_{d_{p}}$
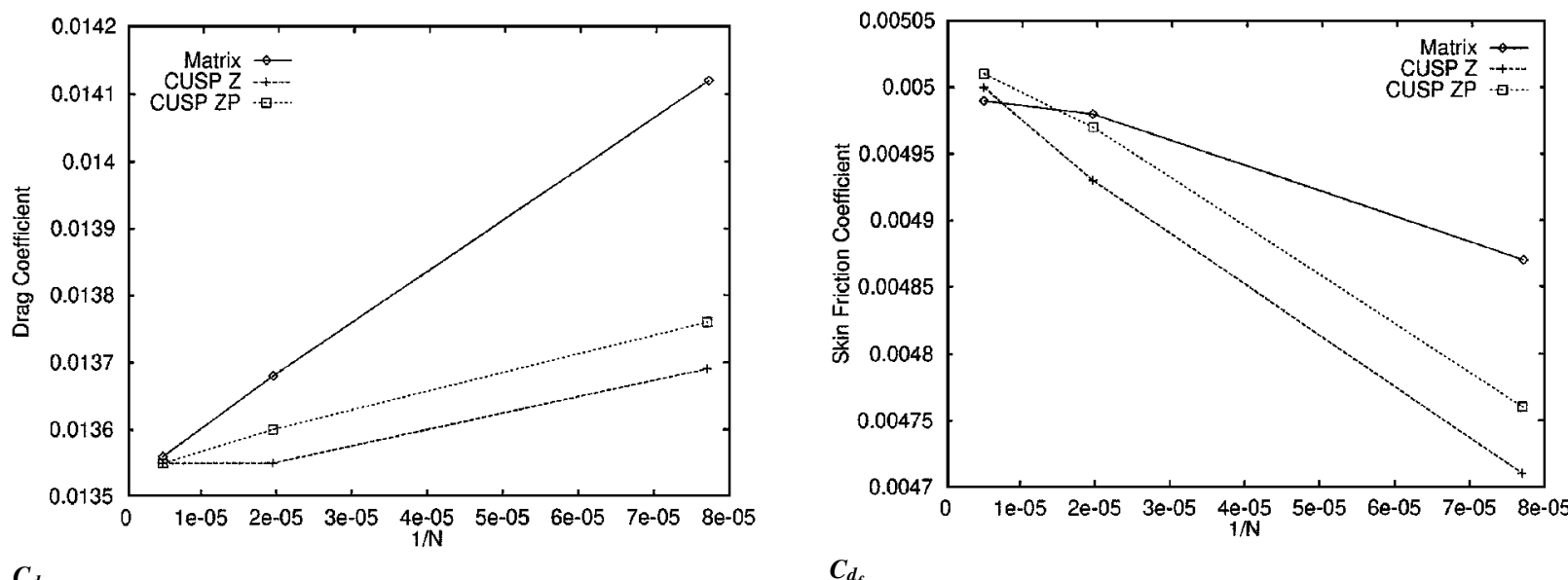

$C_{d}$

Fig. 4 Grid convergence for case 3. 


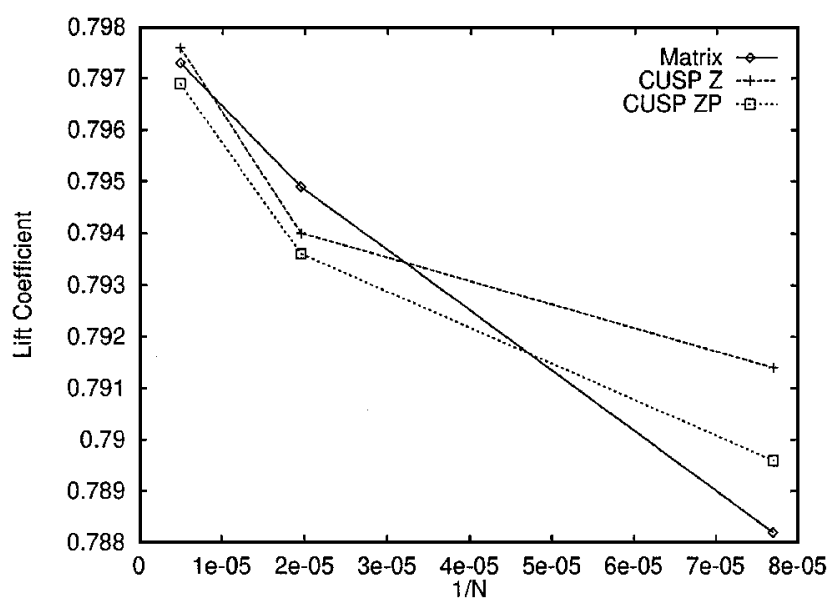

$C_{l}$

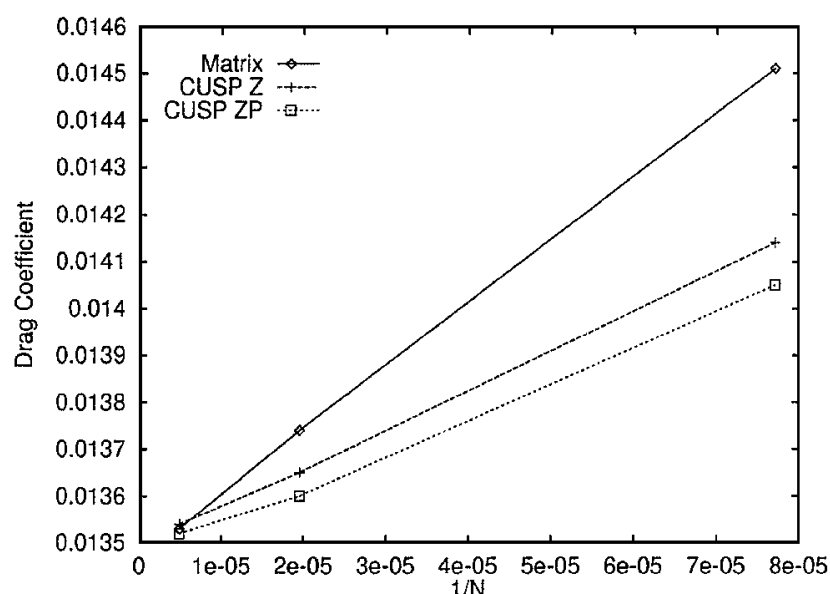

$C_{d}$

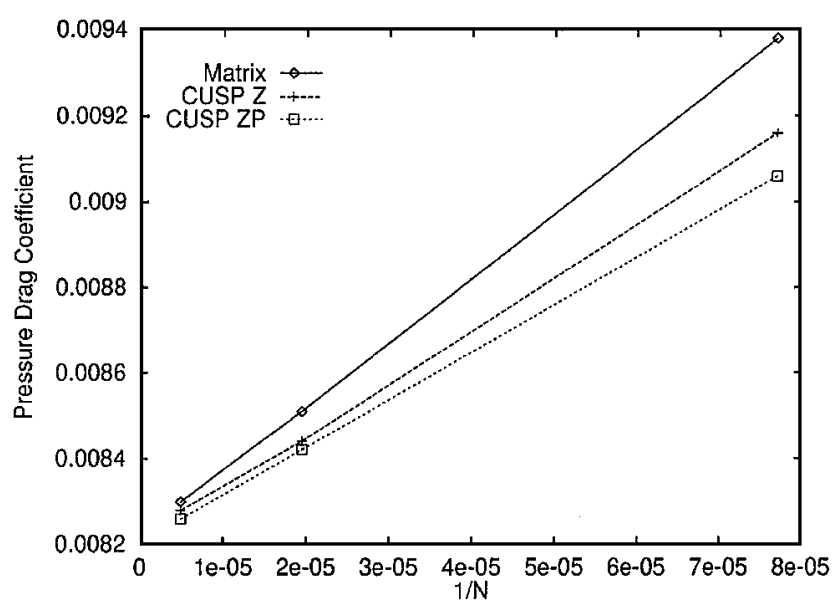

$C_{d_{p}}$

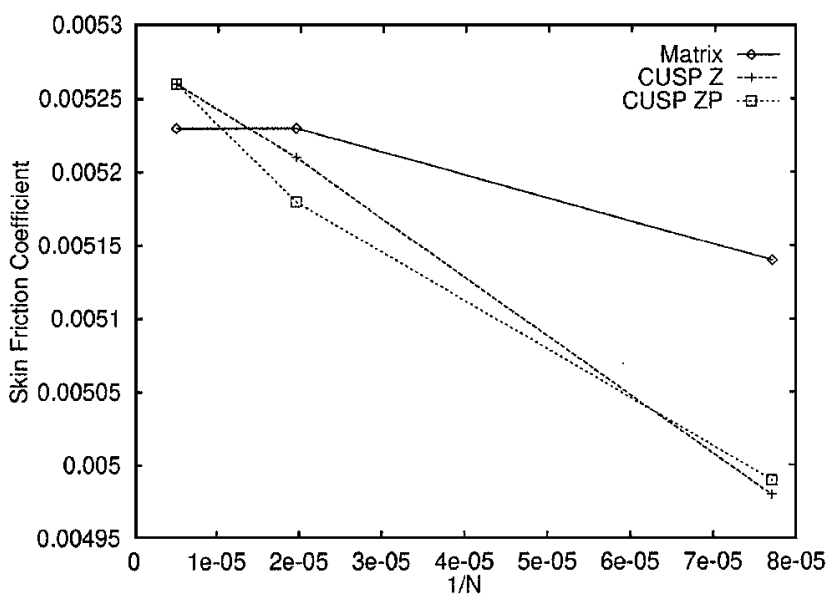

$C_{d_{f}}$

Fig. 5 Grid convergence for case 4.

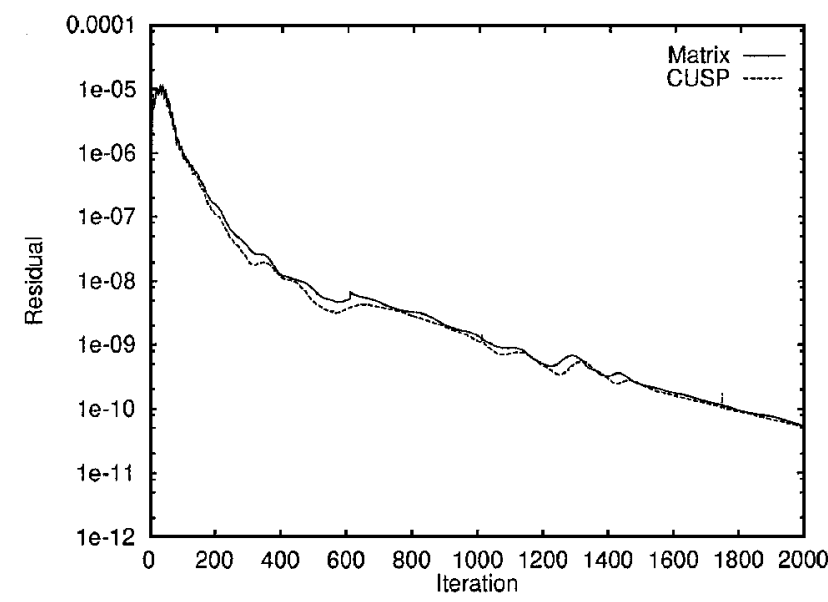

Case 2

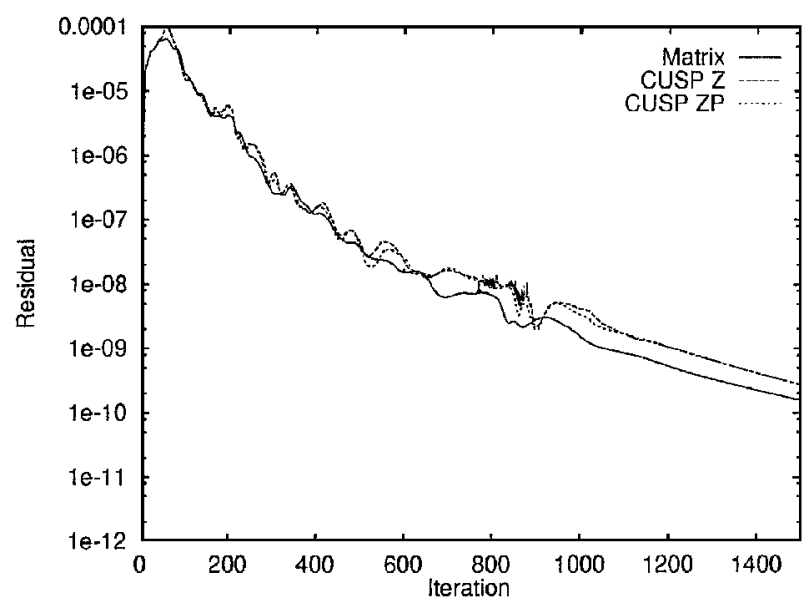

Case 4

Fig. 6 Residual convergence history on grid C.

the convergence is virtually independent of Mach number, leading to significant benefits for Mach numbers below 0.2. Given that the increase in computational expense per iteration associated with the present implementation of local preconditioning is roughly $30 \%$, this translates into substantial savings in overall computational expense for steady computationsat low Mach numbers. Figure 9 shows an improvement in the convergence history of the residual for the preconditionedCUSP scheme at a freestream Mach number of 0.05 .

Although the accuracy of the JST scheme at low Mach numbers is greatly improved through the use of local preconditioning, ${ }^{23}$ it remains inferior to the CUSP scheme. The local preconditioning basically eliminates the speed of sound as an important parameter in the eigenvalues of the flux Jacobian matrices. Thus the sound speed in the locally preconditionedsystem is of the same magnitude as the convective speed, independent of the Mach number. However, the JST numericaldissipation normal to the surface remains much larger than that arising from the preconditionedCUSP scheme because the preconditioned sound speed is much larger than the contravariant velocity component $|V|$ in a boundary layer when the grid is aligned with the flow. 


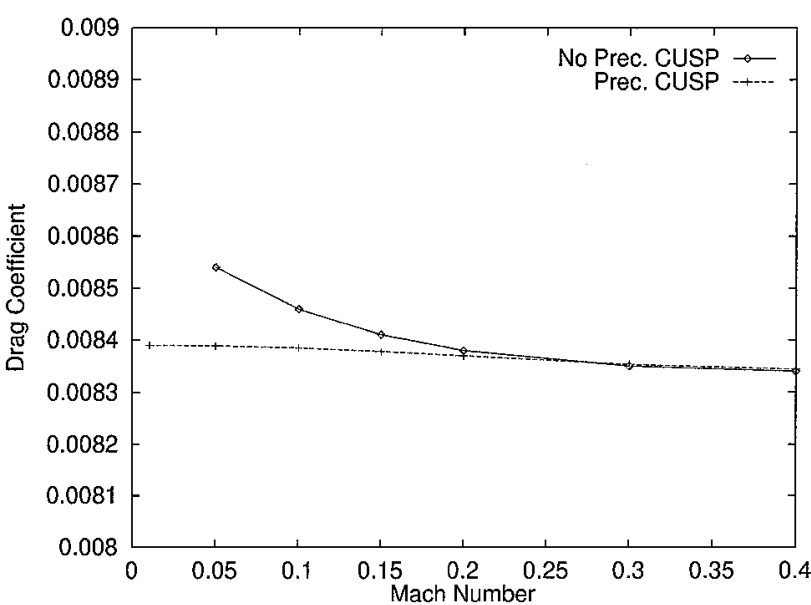

Fig. 7 Computed drag coefficient for case 5.

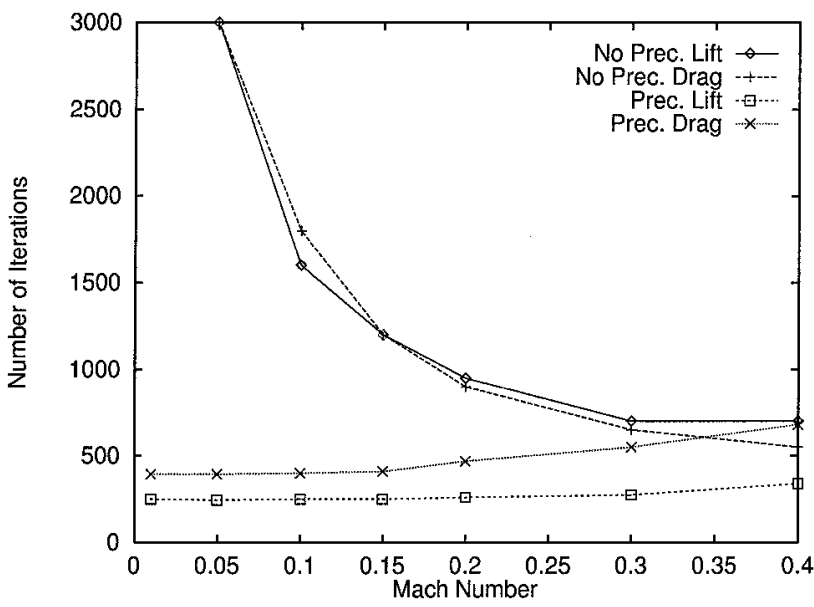

Fig. 8 Convergence of lift and drag for case 5 as a function of the freestream Mach number.

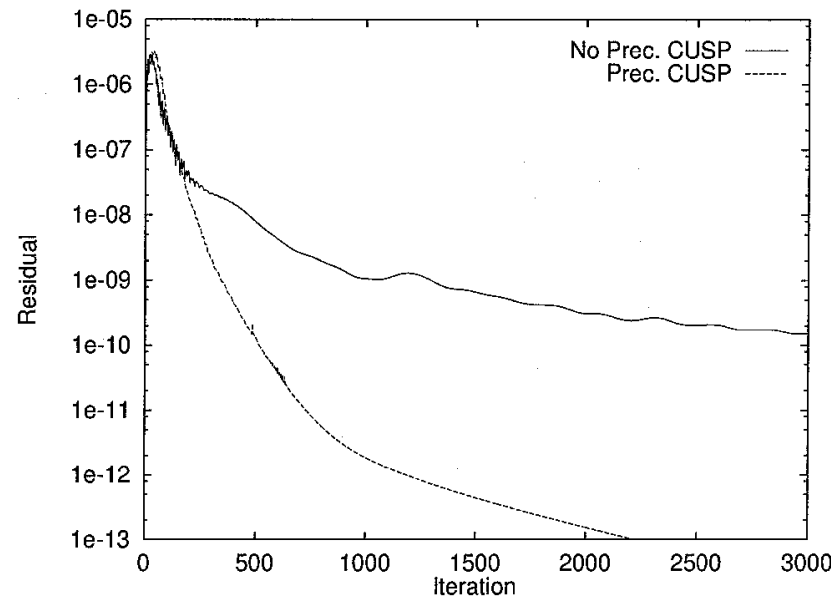

Fig. 9 Residual convergence history for case 5 at $M_{\infty}=0.05$.

\section{Conclusions}

The implementation of a locally preconditioned CUSP scheme has been presented for an approximately factored algorithm in generalized curvilinear coordinates. In addition, an inexpensive flux limiter has been presented and tested. Based on our results for subsonic and transonic airfoil flows, we can draw the following conclusions regarding the use of CUSP for this class of flows:

1) The CUSP scheme provides accuracy that is comparable to the matrix numerical dissipation scheme.

2) When used together with the inexpensive ZP limiter based on pressure, the cost of the CUSP scheme is comparable to that of the original JST scheme.
3) At low Mach numbers the locally preconditioned algorithm produces convergence and accuracy that are virtually independent of Mach number.

Hence the CUSP scheme achieves its goal of providing accuracy comparable to a high-resolution scheme, at least for aerodynamic computations, at a cost similar to that of the JST scheme.

\section{Acknowledgments}

The first author was supported by an Ontario Graduate Scholarship grant of the Government of Ontario. This research was supported by AEA Technology-Advanced Scientific Computing, Ltd., and the Natural Sciences and Engineering Research Council of Canada.

\section{References}

${ }^{1}$ Jameson, A., Schmidt, W., and Turkel, E., "Numerical Solutions of the Euler Equations by Finite Volume Methods Using Runge-Kutta Time Stepping," AIAA Paper 81-1259, June 1981.

${ }^{2}$ Allmaras, S. R., "Contamination of Laminar Boundary Layers by Artificial Dissipation in Navier-Stokes Solutions," Numerical Methods for Fluid Dynamics, edited by M. J. Baines and K. W. Morton, Clarendon, Oxford, 1993.

${ }^{3}$ Frew, K., Zingg, D. W., and De Rango, S., "Artificial Dissipation Schemes for Viscous Airfoil Computations," AIAA Journal, Vol. 36, No. 9 , 1998, pp. 1732-1734.

${ }^{4}$ Roe, P. L., "Approximate Riemann Solvers, Parameter Vectors, and Difference Schemes," Journal of Computational Physics, Vol. 43, No. 2, 1981, pp. 357-372.

${ }^{5}$ Van Leer, B., "Flux Vector Splitting for the Euler Equations," Lecture Notes in Physics, Vol. 170, Springer-Verlag, 1982, pp. 507-512.

${ }^{6}$ Swanson, R. C., and Turkel, E., "On Central-Difference and Upwind Schemes," Journal of Computational Physics, Vol. 101, No. 1, 1992, pp. 292-306.

${ }^{7}$ Harten, A., "High Resolution Schemes for Hyperbolic Conservation Laws," Journal of Computational Physics, Vol. 49, No. 2, 1983, pp. 357 393.

${ }^{8}$ Jameson, A., "Analysis and Design of Numerical Schemes for Gas Dynamics I: Artificial Diffusion, Upwind Biasing, Limiters and Their Effect on Accuracy and Multigrid Convergence," International Journal of Computational Fluid Dynamics, Vol. 4, 1995, pp. 171-218.

${ }^{9}$ Jameson, A., "Analysis and Design of Numerical Schemes for Gas Dynamics II: Artificial Diffusion and Discrete Shock Structure," International Journal of Computational Fluid Dynamics, Vol. 5, No. 1, 1995, 1-38.

${ }^{10}$ Tatsumi, S., Martinelli, L., and Jameson, A., "Flux-Limited Schemes for the Compressible Navier-Stokes Equations," AIAA Journal, Vol. 33, No. 2 1995 , pp. 252-261.

${ }^{11}$ Tatsumi, S., Martinelli, L., and Jameson, A., "A New High Resolution Scheme for Compressible Viscous Flow with Shocks," AIAA Paper 95-0466, Jan. 1995

${ }^{12}$ Jiang, Y. T., and Damodaran, M., "High-ResolutionFinite Volume Computation of Turbulent Transonic Flow past Airfoils," AIAA Journal, Vol. 35, No 7, 1997, pp. 1134-1142.

${ }^{13}$ Sheffer, S. G., Martinelli, L., and Jameson, A., "An Efficient Multigrid Algorithm for Compressible Reactive Flows," Journal of Computational Physics, Vol. 144, No. 2, 1998, pp. 484-516.

${ }^{14}$ Liou, M.-S., and Steffen, J., Jr., "A New Flux Splitting Scheme," Journal of Computational Physics, Vol. 107, No. 1, 1993, pp. 23-39.

${ }^{15}$ Swanson, R. C., Radespiel, R., and Turkel, E., "Comparison of Several Dissipation Algorithms for Central Difference Schemes," AIAA Paper 971945, June 1997.

${ }^{16}$ Zingg, D. W., "Low Mach Number Euler Computations," Canadian Aeronautics and Space Journal, Vol. 36, No. 3, 1990, pp. 146-152.

${ }^{17}$ Volpe, G., "On the Use and Accuracy of Compressible Flow Codes at Low Mach Numbers,” AIAA Paper 91-1662, June 1991.

${ }^{18}$ Turkel, E., "Review of Preconditioning Methods for Fluid Dynamics," Applied Numerical Mathematics, Vol. 12, 1993, pp. 257-284.

${ }^{19}$ Choi, Y. H., and Merkle, C. L., "The Application of Preconditioning in Viscous Flows," Journal of Computational Physics, Vol. 105, No. 2, 1993 pp. 207-233.

${ }^{20}$ Turkel, E., "Preconditioned Methods for Solving the Incompressible and Low Speed Compressible Equations," Journal of Computational Physics, Vol. 72, No. 2, 1987, pp. 277-298.

${ }^{21}$ Van Leer, B., Lee, W. T., and Roe, P., "Characteristic Time-Stepping or Local Preconditioning of the Euler Equations," AIAA Paper 91-1552, June 1991.

${ }^{22}$ Turkel, E., Fiterman, A., and Van Leer, B., "Preconditioning and the Limit of the Compressible to the Incompressible Flow Equations for Finite Difference Schemes," Frontiers of Computational Fluid Dynamics 1994, edited by D. A. Caughey and M. Hafez, Wiley, New York, 1994, pp. 215 234 
${ }^{23}$ Unrau, D., and Zingg, D. W., "Viscous Airfoil Computations Using Local Preconditioning," AIAA Paper 96-2088, June 1996.

${ }^{24}$ Weiss, J. M., and Smith, W. A., "Preconditioning Applied to Variable and Constant Density Flows," AIAA Journal, Vol. 33, No. 11, 1997, pp. 2050-2057.

${ }^{25}$ Tweedt, D. L., Chima, R. V., and Turkel, E., "Preconditioning for $\mathrm{Nu}$ merical Simulation of Low Mach Number Three-Dimensional Viscous Turbomachinery Flows," AIAA Paper 97-1828, June 1997.

${ }^{26}$ Edwards, J. R., and Liou, M.-S., "Low-Diffusion Flux-Splitting Methods for Flows at All Speeds," AIAA Paper 97-1862, June 1997.

${ }^{27}$ Pulliam, T. H., "Efficient SolutionMethods for the Navier-Stokes Equations," Lecture Notes for the von Kármán Inst. for Fluid Dynamics Lecture Series: Numerical Techniques for Viscous Flow Computation in Turbomachinery Bladings, von Kármán Inst. for Fluid Dynamics, Brussels, Belgium, Jan. 1986.

${ }^{28}$ Turkel, E., Radespiel, R., and Kroll, N., “Assessment of Precondition- ing Methods for Multidimensional Aerodynamics," Computers and Fluids, Vol. 26, No. 6, 1997, pp. 613-634.

${ }^{29}$ Jorgenson, P., and Turkel, E., "Central Difference TVD Schemes for Time Dependent and Steady State Problems," Journal of Computational Physics, Vol. 107, No. 2, 1993, pp. 297-308.

${ }^{30}$ Nemec, M., "Aerodynamic Computations Using the Convective Upstream Split Pressure Scheme with Local Preconditioning," M.S. Thesis, Dept. of Aerospace Science and Engineering, Univ. of Toronto, Toronto, ON, Canada, April 1998.

${ }^{31}$ Venkatakrishnan, V., "Convergence to Steady State Solutions of the Euler Equations on Unstructured Grids with Limiters," Journal of Computational Physics, Vol. 118, No. 1, 1995, pp. 120-130. 